\title{
Rapidity evolution of Wilson lines at the next-to-leading order: Balitsky-JIMWLK equation at NLO
}

\author{
Giovanni Antonio Chirilli \\ Department of Physics, The Ohio State University, Columbus, OH 43210, USA
}

\begin{abstract}
Scattering amplitudes of proton-Nucleus or Nucleus-Nucleus collisions at high-energy are described by matrix elements of Wilson line operators - infinite gauge factors ordered along the straight line trajectories of the fast moving particles. The energy dependence of such amplitudes is described by the evolution equation of Wilson lines with respect to the rapidity parameter - the BalitskyJIMWLK evolution equation. Most of the current phenomenology of high-energy and high-density QCD is based on the leadingorder evolution equation with only running coupling corrections. I will present the derivation of the Balitsky-JIMWLK evolution equation at the next-to-leading order.
\end{abstract}

Keywords: perturbative QCD, small-x evolution, heavy-ion collisions

\section{Introduction}

It is very well known that the main degrees of freedom in high energy scatterings are Wilson line operators: infinite gauge factors ordered along the straight lines of the fast moving particles. The notion of Wilson line can be traced back to quantum mechanics. If we consider a particle propagating at very high speed through an external potential in the high-energy limit, the wave function of the particle is modified by multiplying the wave function of the particle in free space by a phase factor given by the exponential of the external potential ordered along the line parallel to the particle's velocity. In quantum electrodynamics the wave function of the particle is modified in the same way, but this time it is the gauge field $A^{\mu}(x)$ that enters in the exponential phase factor.

In QCD, since the gauge field is a matrix valued function, the phase factor is a path-ordered exponential along the trajectory of the particle

$$
U\left(x_{\perp}\right)=1+i g \int_{-\infty}^{\infty} d u A_{\bullet}\left(u p_{1}+x_{\perp}\right)+(i g)^{2} \int_{-\infty}^{+\infty} d u \int_{-\infty}^{u} d v A_{\bullet}\left(u p_{1}+x_{\perp}\right) A_{\bullet}\left(v p_{1}+x_{\perp}\right)+\ldots
$$

Here we used the notation $x_{\bullet}=\sqrt{\frac{s}{2}} x^{-}$and $x_{*}=\sqrt{\frac{s}{2}} x^{+}$with $x^{+}$and $x^{-}$light-cone coordinates defined as $x^{ \pm}=\frac{x^{0} \pm x^{3}}{\sqrt{2}}$; $p_{1}^{\mu}$ and $p_{2}^{\mu}$ are ligh-cone vectors such that $p_{1}^{2}=p_{2}^{2}=0$ and $p_{1} \cdot p_{2}=\frac{s}{2}$ with $s$ the Mandelstam variable for the center-of-mass energy.

As it has been shown in Refs $[1,2]$, a useful tool to calculate scattering amplitudes at high energy is the Operator Product Expansion (OPE) in Wilson lines; the scattering amplitude is written as a convolution of coefficient functions and matrix elements of Wilson lines ordered along the particles' velocities, while the energy dependence of the amplitudes is encoded in the evolution equations in rapidity of the Wilson lines. In Deep Inelastic Scattering (DIS) at high energy the virtual photon emitted by the lepton long before scattering off an hadronic target, splits into a quark 
and anti-quark pair. The propagation of the color dipole through the hadronic target, made mainly by gluon field, is given by a scattering amplitude proportional to two Wilson lines. Thus, the high-energy DIS scattering amplitude is written as a convolution of a coefficient function (the photon impact factor now known at NLO [6, 7]) and a matrix element of a color dipole. The evolution equation of the color dipole is the first of the Balitsky hierarchy of equations which, in the large $N_{c}$ approximation, reduces to the Balitsky-Kovchegov equation[1, 3] (for a review see Ref. [5]).

In proton-Nucleus or Nucleus-Nucleus collisions the scattering amplitudes are made of several Wilson lines and the corresponding evolution equation is known as the Balitsky-JIMWLK evolution equation[1, 4].

\section{Leading Order Evolution Equation}

As stated in the introduction, the energy dependence of the high-energy scattering amplitude is encoded in the evolution of matrix elements made of Wilson lines. In order to obtain the evolution equation we will use the background field method.

Let us suppose that $O^{\eta_{1}}$ is an operator made of several Wilson lines with rapidity $\eta_{1}$, and if we indicate with $|B\rangle$ the target state, then the scattering amplitude is proportional to $\left\langle B\left|O^{\eta_{1}}\right| B\right\rangle$. In order to obtain the evolution equation we do not need to know the details of the target so, we evaluate the operator $O^{\eta_{1}}$ in the background of an external gluonic field (at high energy the gluon degrees of freedom dominate over the sea and valence quark degrees of freedom).

The second step is to introduce a rapidity divide $\eta_{2}$ : fields with rapidity up to $\eta_{2}$ are treated as classical fields, while fields with rapidity $\eta_{1}>\eta>\eta_{2}$ are the quantum fluctuations that will be integrated out. Formally, the separation of the correlation function in classical and quantum fields may be written as follows

$$
\left\langle O^{\eta_{1}}\right\rangle_{A} \rightarrow\left\langle O^{\prime \eta_{2}} \otimes O^{\prime \eta_{1}}\right\rangle_{A}
$$

where the subscript $A$ indicates that the matrix elements are evaluated in the background of the gluonic external field. In principle, after the separation in classical and quantum fields, the operator may change, and this is indicated with a prime on the operator $O$ in Eq. (2). However, since particles with different rapidity perceive each other as Wilson lines, the operators obtained after splitting the fields in classical and quantum components are still Wilson lines.

The result of the integration of the matrix element on the right-hand-side (RHS) of Eq. (2) over the quantum fields, is the kernel of the one loop evolution equation times the matrix element of the operator made of the classical fields i.e. with rapidity parallel to $\eta_{2}$, and times the infinitesimal step in rapidity $\Delta \eta=\eta_{2}-\eta_{1}$. The evolution equation of the $O$ operator with respect to rapidity is

$$
\left\langle O^{\eta_{1}}\right\rangle_{A}=\alpha_{s}\left(\eta_{1}-\eta_{2}\right) K_{\mathrm{evol}} \otimes\left\langle O^{\prime \eta_{2}}\right\rangle_{A}
$$

The resulting evolution equation obtained following the procedure just described, can be linear or non-linear. This can formally be written as

- Linear case $O^{\eta_{1}}=\alpha_{s} \Delta \eta K_{\mathrm{evol}} \otimes O^{\eta_{2}}$

- Non-linear case $O^{\eta_{1}}=\alpha_{s} \Delta \eta K_{\text {evol }} \otimes\left\{O^{\eta_{2}} O^{\eta_{2}}\right\}$

If we consider, for example, the evolution of a matrix element made of one Wilson line, the Feynman diagrams of the evolution equation are given in Fig. (2). The red strip in the figure represents the background field in the spectator frame: the external field is highly boosted, and it gets contracted in the direction of the boost and time-dilated. Thus, the propagation of the particle is in the background of a shock-wave external field. The evolution equation for one
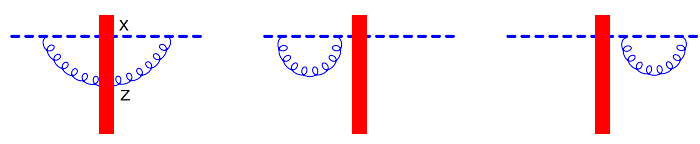

Figure 1. Feynman diagrams for one loop evolution of one Wilson lines. 
Wilson line is

$$
\left\langle\left\{U_{x}^{\eta_{1}}\right\}_{i j}\right\rangle_{A}=\frac{\alpha_{s}}{2 \pi^{2}} \Delta \eta \int \frac{d^{2} z}{(x-z)_{\perp}^{2}}\left[\left\langle\operatorname{tr}\left\{U_{x}^{\eta_{2}} U_{z}^{\eta_{2} \dagger}\right\}\left\{U_{z}^{\eta_{2}}\right\}_{i j}\right\rangle_{A}-\left\langle\frac{1}{N_{c}}\left\{U_{x}^{\eta_{2}}\right\}_{i j}\right\rangle_{A}\right]
$$

where we have used the short-hand notation $U_{x} \equiv U\left(x_{\perp}\right)$, and the color index $i, j=1,2,3$. The kernel of the evolution in this case is $K(x, z)=\frac{1}{(x-z)_{\perp}^{2}}$, and the equation is clearly non-linear. Before the one-loop evolution, we have one Wilson line with rapidity $\eta_{1}$ corresponding to the propagation of one quark in the background of a shock-wave. At one loop order, instead, we have a quark and a gluon propagating in the shock-wave.

In DIS at high-energy, the scattering amplitude is proportional to $\mathcal{U}\left(x_{\perp}, y_{\perp}\right)=1-\frac{1}{N_{c}} \operatorname{tr}\left\{U\left(x_{\perp}\right) U^{\dagger}\left(y_{\perp}\right)\right\}$. To get the evolution equation of the color-dipole we need the evolution equation of the operator $U_{x}$ and $U_{y}^{\dagger}$, and also the evolution equation for one loop correction between $U_{x}$ and $U_{y}^{\dagger}$ i.e pairwise interactions. Thus, the operator $\frac{d}{d \eta}$ of the evolution equation does not follow the Leibniz rule for derivative of product of function. This is represented by the Feynman diagrams in Fig. (2) and the corresponding evolution equation is

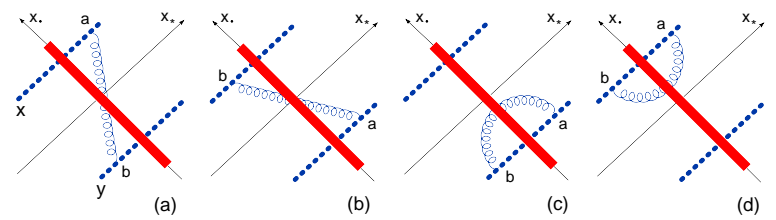

Figure 2. Feynamn diagram for the LO evolution equation of a color-dipole. We omitted the virtual correction diagrams.

$$
\frac{d}{d \eta} \hat{\mathcal{U}}(x, y)=\frac{\alpha_{s} N_{c}}{2 \pi^{2}} \int \frac{d^{2} z(x-y)^{2}}{(x-z)^{2}(y-z)^{2}}\{\hat{\mathcal{U}}(x, z)+\hat{\mathcal{U}}(z, y)-\hat{\mathcal{U}}(x, y)-\hat{\mathcal{U}}(x, z) \hat{\mathcal{U}}(z, y)\}
$$

Equation (4) is the LO Balitsky evolution equation[1] for color dipole. When the non-linear term operator $\langle\hat{\mathcal{U}}(x, z) \hat{\mathcal{U}}(z, y)\rangle$ factorizes at large $N_{c}$ as $\langle\hat{\mathcal{U}}(x, z)\rangle\langle\hat{\mathcal{U}}(z, y)\rangle$, Eq. (4) becomes the Balitsky-Kovchegov equation[1, 3]. The linear terms in Eq. (4) correspond to the BFKL evolution equation obtained in perturbative QCD in the leading$\log$ resummation $\alpha \ll 1$ and $\alpha_{s} \eta \sim 1$; while the non-linear term appears because of the semi-classical approach where the new resummation parameter is $\alpha_{s}^{2} A^{1 / 3}$ with $A$ being the atomic number in the case of DIS off a nuclear target. The BFKL equation is known to violate unitarity, but the non linear term in Eq. (4) restores unitarity.

In order to get the LO evolution equation for trace of any number of Wilson lines or product of any number of Wilson lines, which would correspond to the Balitsky-JIMWLK evolution equation[1, 4], one needs to obtain the evolution equation for the following operators: $\left\{U_{x}^{\dagger \eta_{1}}\right\}_{i j},\left\{U_{x}^{\eta_{1}} U_{y}^{\eta_{1}}\right\}_{i j},\left\{U_{x}^{\eta_{1}} U_{y}^{\dagger \eta_{1}}\right\}_{i j},\left\{U_{x}^{\dagger \eta_{1}} U_{y}^{\dagger \eta_{1}}\right\}_{i j}$. Thus, one obtains a set of five evolution equations that can be used to calculate the evolution equation of operators with any trace of Wilson lines. For example, to calculate the evolution equation of a four Wilson lines operator $\operatorname{tr}\left\{U_{x} U_{y}^{\dagger} U_{w} U_{z}^{\dagger}\right\}$ one has to sum the evolution of each single Wilson line using the evolution equation like Eq. (2), and similar evolution equations for each paring.

We would like to extend these rules at the next-to-leading order (NLO) in order to obtain the evolution equations of matrix elements of operators with any trace of Wilson lines at NLO.

\section{Next-to-leading order evolution equation}

In order to obtain the evolution equation of operators with any trace of Wilson lines (or also product of Wilson lines) at the next to leading order, one has to calculate a similar set of evolution equations at NLO. At NLO, however, we may have not only the evolution of single Wilson lines and of two connected Wilson lines but also evolution of triple Wilson lines interaction (see Fig. 3 e) and f)). The diagrams contributing to the NLO B-JIMWLK with three Wilson lines were calculated in Ref. [9]. In Ref. [10] we have calculated the full Balitsky hierarchy at NLO, confirming also the result obtained in Ref. [9]. The NLO JIMWLK Hamiltonian[11], on the other hand, was obtained using the NLO BK equation calculated in Ref. [12] and the evolution with three connected Wilson lines of Ref. [9]. 

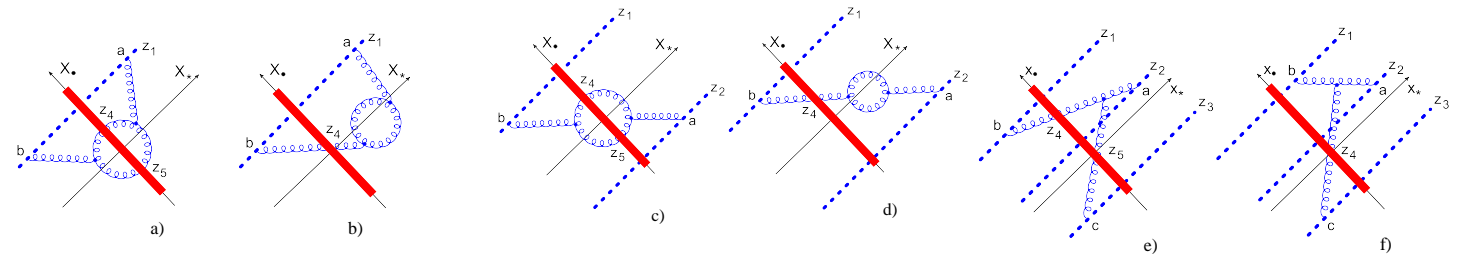

Figure 3. Sample of Feynman diagrams contributing to the NLO B-JIMWLK evolution equation.

We will present here only the one-particle interaction ("gluon reggeization" term) at NLO

$$
\begin{aligned}
& \frac{d}{d \eta}\left(U_{1}\right)_{i j}=\frac{\alpha_{s}^{2}}{8 \pi^{4}} \int \frac{d^{2} z_{4} d^{2} z_{5}}{z_{45}^{2}}\left\{U _ { 4 } ^ { d d ^ { \prime } } ( U _ { 5 } ^ { e e ^ { \prime } } - U _ { 4 } ^ { e e ^ { \prime } } ) \left(\left[2 I_{1}-\frac{4}{z_{45}^{2}}\right] f^{a d e} f^{b d^{\prime} e^{\prime}}\left(t^{a} U_{1} t^{b}\right)_{i j}+\frac{\left(z_{14}, z_{15}\right)}{z_{14}^{2} z_{15}^{2}} \ln \frac{z_{14}^{2}}{z_{15}^{2}}\right.\right. \\
& \left.\left.\times\left[i f^{a d^{\prime} e^{\prime}}\left(\left\{t^{d}, t^{e}\right\} U_{1} t^{a}\right)_{i j}-i f^{a d e}\left(t^{a} U_{1}\left\{t^{d^{\prime}}, t^{e^{\prime}}\right\}\right)_{i j}\right]\right)\right\} \\
& +\frac{\alpha_{s}^{2} N_{c}}{4 \pi^{3}} \int d^{2} z_{4} z_{14}^{2}\left(U_{4}^{a b}-U_{1}^{a b}\right)\left(t^{a} U_{1} t^{b}\right)_{i j}\left\{\left[\frac{11}{3} \ln z_{14}^{2} \mu^{2}+\frac{67}{9}-\frac{\pi^{2}}{3}\right]\right\}
\end{aligned}
$$

where we have used the short-hand notation $U_{1} \equiv U\left(z_{1 \perp}\right)$ etc., and the explicit form of the function $I_{1} \equiv I\left(z_{1 \perp}, z_{4 \perp}, z_{5 \perp}\right)$ can be found in Ref. [10]. In Eq. (5) we have omitted the quark-loop contributions. The pairwise interaction and the triple interaction at NLO can be found in Ref. [10].

\section{Conclusions}

We have reported the result of the NLO Balitsky-JIMWLK evolution equation and given a brief pedagogical introduction to the background field method. The factorization in rapidity of scattering amplitudes using the OPE at high-energy in Wilson lines is a successful tool for the study of processes at high energy. The BK equation is a large $N_{c}$ limit of the first of the Balitsky hierarchy equations. In order to generalize this result to NLO we have to consider the triple interactions besides the self-energy interactions and the pairwise interactions. For simplicity, we have presented only the evolution equation for the self-energy interaction at NLO, while the NLO pairwise interactions and the triple interactions can be found in Ref. [10].

This material is based upon work supported by the U.S. Department of Energy, Office of Science, Office of Nuclear Physics under Award Number DE-SC0004286.

\section{References}

[1] I. Balitsky, Nucl. Phys. B 463, 99 (1996) [hep-ph/9509348].

[2] I. Balitsky and G. A. Chirilli, Phys. Lett. B 687, 204 (2010) [arXiv:0911.5192 [hep-ph]].

[3] Y. V. Kovchegov, Phys. Rev. D 60, 034008 (1999) [hep-ph/9901281].

[4] J. Jalilian Marian, A. Kovner, A.Leonidov and H. Weigert, Nucl. Phys. B504, 415 (1997), Phys. Rev. D59, 014014 (1999); J. Jalilian Marian, A. Kovner and H. Weigert, Phys. Rev. D59, 014015 (1999); A. Kovner and J.G. Milhano, Phys. Rev. D61, 014012 (2000); A. Kovner, J.G. Milhano and H. Weigert, Phys. Rev. D62, 114005 (2000); H. Weigert, Nucl. Phys. A703, 823 (2002); E.Iancu, A. Leonidov and L. McLerran, Nucl. Phys. A692, 583 (2001), Phys. Lett. B510, 133 (2001); E. Ferreiro, E. Iancu, A. Leonidov, L. McLerran, Nucl. Phys. A703, 489 (2002).

[5] I. Balitsky, In *Shifman, M. (ed.): At the frontier of particle physics, vol. 2* 1237-1342 [hep-ph/0101042].

[6] I. Balitsky and G. A. Chirilli, Phys. Rev. D 87, 014013 (2013) [arXiv:1207.3844 [hep-ph]].

[7] I. Balitsky and G. A. Chirilli, Phys. Rev. D 83, 031502 (2011) [arXiv:1009.4729 [hep-ph]].

[8] I. Balitsky and G. A. Chirilli, Nucl. Phys. B 822, 45 (2009) [arXiv:0903.5326 [hep-ph]].

[9] A. V. Grabovsky, JHEP 1309, 141 (2013) [arXiv:1307.5414 [hep-ph]].

[10] I. Balitsky and G. A. Chirilli, Phys. Rev. D 88, 111501 (2013) [arXiv:1309.7644 [hep-ph]].

[11] A. Kovner, M. Lublinsky and Y. Mulian, Phys. Rev. D 89, 061704 (2014) [arXiv:1310.0378 [hep-ph]].

[12] I. Balitsky and G. A. Chirilli, Phys. Rev. D 77, 014019 (2008) [arXiv:0710.4330 [hep-ph]]. 